PAPER

\title{
Parametric speaker consisting of small number of transducers with sonic crystal waveguide
}

\author{
Jun Kuroda* and Yasuhiro Oikawa \\ Department of Intermedia Art and Science, Waseda University, \\ 3-4-1 Ohkubo, Shinjuku-ku, Tokyo, 169-8555 Japan
}

(Received 15 October 2019, Accepted for publication 15 May 2020)

\begin{abstract}
In this paper, we discuss methods of designing parametric speakers consisting of a small number of transducers. The developed parametric speaker unit includes peripheral acoustic structures, such as radial cones and a waveguide comprising a sonic crystal. The purpose of this study is to fabricate small parametric speakers that can be installed in various equipment. To achieve this purpose, it is essential to minimize the sizes and numbers of transducers. Parametric speakers must fulfill two goals: (1) high sound pressure levels of ultrasonic waves and (2) narrow directivities of ultrasonic waves. These two design goals are accomplished by two measures: (1) two close resonant frequencies and resonant mode control function and (2) an external adapter to obtain narrow directivities and high sound pressure. The first measure was achieved equipping the piezoelectric transducer with double-linked diaphragms and two radial cones. The second measure was embodied by the waveguide consisting of a sonic crystal, which was named the loop horn in this paper. Details of the design and experimental data of a four-transducer parametric speaker unit including the loop horn were presented.
\end{abstract}

Keywords: Piezoelectric transducer, Ultrasonic wave, Parametric speaker, Sonic crystal

PACS number: 43.38.Fx, 43.40.At [doi:10.1250/ast.41.865]

\section{INTRODUCTION}

Parametric speaker arrays have been studied for speech privacy systems since Westervelt presented his paper on parametric acoustic arrays (in 1963) [1-4]. The principle of parametric speakers is based on the nonlinear phenomenon of sound waves [5,6]. Moreover, the generated virtual sound sources and the sound demodulated by parametric speakers have also been well studied [7-9]. The parametric speaker was first invented by Yoneyama et al. in 1983 as an "audio spotlight" [3]. Parametric speakers provide narrow directional audible sound using modulated ultrasonic waves. They have many expected applications, such as, in conveying information in museums and offices, and in voice communication using smart devices. For shortrange voice communication using smart electric devices and their peripheral gadgets, parametric speakers are expected to provide privacy protection of voice communication. In this paper, we discuss a parametric speaker unit that can be used for short-range voice communication in personal spaces and offices.

*e-mail: jun-kuroda@suou.waseda.jp
The important issues limiting the use of parametric speakers for short-range voice communication are manufacturability and maintainability. It is difficult to control the quality of piezoelectric transducers in mass production when many piezoelectric transducers are used in one unit of a parametric speaker product.

To solve these two problems in actual mass production, it is necessary to reduce the number of piezoelectric transducers used in one parametric speaker. To reduce the number of transducers, we must obtain a parametric speaker satisfying the following two conditions,

A. Wide available bandwidth around resonant peaks and high sound pressure level of radiated ultrasonic waves B. Narrow directivities of ultrasonic fields

The two target characteristics denoted above are obtained by two design methods.

1. Obtain two close resonant frequencies and a mode control function

2. Design an acoustic waveguide as an external attachment of a transducer unit

The first method can be achieved using the doublelinked structure shown in our previous papers $[10,11]$. The developed transducer has two diaphragms linked by three 
cylindrical rods and a resonant mode control function. With the developed structure and function, we obtained a stable and functional transducer that can increase the available width of the resonant peaks. The second method can be obtained using a waveguide, which is a special horn for ultrasonic waves. Original horns, such as conical horns and exponential horns are inappropriate for ultrasonic waves because the wavelength of an ultrasonic wave is much smaller than the dimensions of the horns, which means that the assumption of Webster's horn equation is not satisfied. We proposed methods of fabricating a parametric speaker consisting of a small number of transducers having a high sound pressure level and narrow radiation patterns of demodulated sounds.

\section{DESIGN OF TRANSDUCER}

\subsection{Basic Theory of Transducer}

In our previous paper, we described the basic structure of a developed transducer, i.e., the transducer with the double-linked diaphragms and the function of resonant mode control using two input voltage channels. Figures 1(a) and 1(b) show the basic structure and configuration of the electrical connection of the developed transducer, respectively. This transducer was developed to study the mode control function. It has two close resonant frequencies and modes that can be controlled by adjusting the phase difference between two voltage signals applied to two piezoelectric elements. As shown in Fig. 1(a), the developed transducer has three rods that connect two diaphragms. Two piezoelectric elements are adhered onto the two diaphragms. Two voltage signals are input to two piezoelectric elements, as shown in Fig. 1(b). To improve the efficiency of the electroacoustic conversion of the transducer, we should add radial cones, which act as a type of displacement magnification mechanism. In general, piezoelectric ultrasonic transducers have a radial cone [12].

A radial cone is usually made of a light metal, such as a plate of aluminum alloy. The aluminum alloy plate is deformed into a cone shape using a turret punch press and finally it is adhered to a diaphragm using adhesives. Note
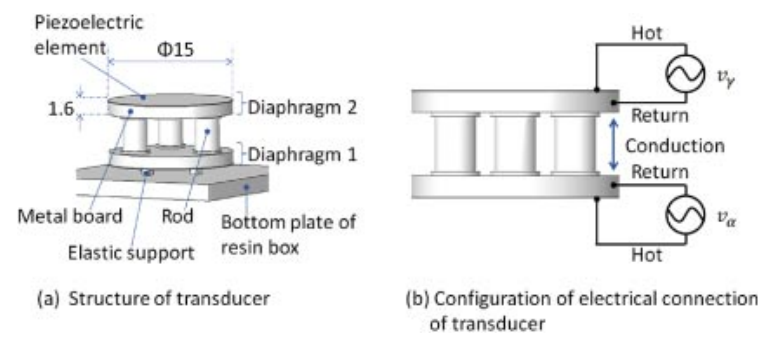

(a) Structure of transducer that the theoretical analysis of radial cones requires knowledge related to complicated shell vibration $[13,14]$.

Because our developed transducer has two diaphragms, we must consider the following two design conditions Requirement 1 . A dummy horn is necessary to maintain the function of the resonant mode control.

Requirement 2. The contact part between the two diaphragms and radial cones must be stable.

Requirement 1 arises because of the function of the resonant mode control of the developed transducer, i.e., to maintain the balance between diaphragms 1 and 2 of the developed transducer, a dummy cone is necessary. Requirement 2 is related to the stability and strength of the adhered part between the radial cones and diaphragms. If the bonding area is insufficient, the vibration force transmitted to the radial cones and the efficiency of the displacement magnification will decrease.

The two requirements can be discussed using a mechanical equivalent circuit. Figures 2 and 3 show the developed transducer with radial cones and its mechanical equivalent circuit, respectively. The mechanical equivalent circuit shown in Fig. 3 is expressed by four ordinary differential equations (ODEs) as

$$
\begin{aligned}
& m_{\alpha} \ddot{\Phi}_{\alpha}+r_{\alpha} \dot{\Phi}_{\alpha}+s_{\alpha} \Phi_{\alpha} \\
& +\left[s_{\alpha c}\left(\zeta_{\alpha c} \Phi_{\alpha}-\zeta_{c \alpha} \Phi_{c d}\right)+r_{\alpha c}\left(\zeta_{\alpha c} \dot{\Phi}_{\alpha}-\zeta_{c \alpha} \dot{\Phi}_{c d}\right)\right] \\
& +\left[s_{\beta}\left(\zeta_{\alpha} \Phi_{\alpha}-\zeta_{\gamma} \Phi_{\gamma}\right)+r_{\beta}\left(\zeta_{\alpha} \dot{\Phi}_{\alpha}-\zeta_{\gamma} \dot{\Phi}_{\gamma}\right)\right]=F_{\alpha},
\end{aligned}
$$

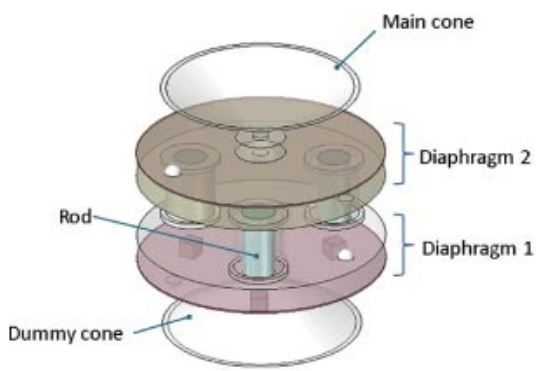

Fig. 2 Schematic of the developed transducer having the double-linked diaphragms and the radial cones.

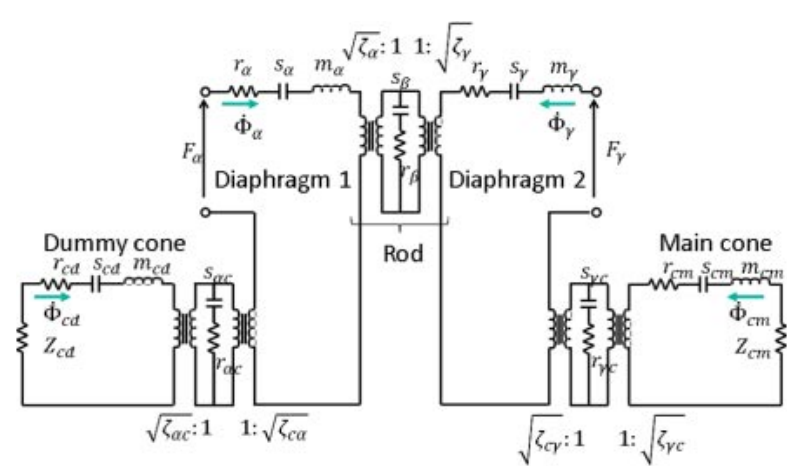

Fig. 3 Schematic of the mechanical equivalent circuit of the piezoelectric transducer with two radial cones.
Fig. 1 Schematic of the basic structure of developed transducer having double-linked diaphragms and the mode control function. 


$$
\begin{aligned}
& m_{c d} \ddot{\Phi}_{c d}+r_{c d} \dot{\Phi}_{c d}+s_{c d} \Phi_{c d}+Z_{c d} \dot{\Phi}_{c d} \\
& \quad-\left[s_{\alpha c}\left(\zeta_{\alpha c} \Phi_{\alpha}-\zeta_{c \alpha} \Phi_{c d}\right)+r_{\alpha c}\left(\zeta_{\alpha c} \dot{\Phi}_{\alpha}-\zeta_{c \alpha} \dot{\Phi}_{c d}\right)\right]=0
\end{aligned}
$$

$$
\begin{aligned}
& m_{\gamma} \ddot{\Phi}_{\gamma}+r_{\gamma} \dot{\Phi}_{\gamma}+s_{\gamma} \Phi_{\gamma} \\
& \quad+\left[s_{\gamma c}\left(\zeta_{\gamma c} \Phi_{\gamma}-\zeta_{c \gamma} \Phi_{c m}\right)+r_{\gamma c}\left(\zeta_{\gamma c} \dot{\Phi}_{\gamma}-\zeta_{c \gamma} \dot{\Phi}_{c m}\right)\right] \\
& \quad-\left[s_{\beta}\left(\zeta_{\alpha} \Phi_{\alpha}-\zeta_{\gamma} \Phi_{\gamma}\right)+r_{\beta}\left(\zeta_{\alpha} \dot{\Phi}_{\alpha}-\zeta_{\gamma} \dot{\Phi}_{\gamma}\right)\right]=F_{\gamma}
\end{aligned}
$$

and

$$
\begin{aligned}
& m_{c m} \ddot{\Phi}_{c m}+r_{c m} \dot{\Phi}_{c m}+s_{c m} \Phi_{c m}+Z_{c m} \dot{\Phi}_{c m} \\
& \quad-\left[s_{\gamma c}\left(\zeta_{\gamma c} \Phi_{\gamma}-\zeta_{c \gamma} \Phi_{c m}\right)+r_{\gamma c}\left(\zeta_{\gamma c} \dot{\Phi}_{\gamma}-\zeta_{c \gamma} \dot{\Phi}_{c m}\right)\right]=0,
\end{aligned}
$$

where $\Phi_{\alpha}, \Phi_{\gamma}, \Phi_{c m}$, and $\Phi_{c d}$ are the equivalent vibration displacement of diaphragms 1 and 2 , and the main and dummy cones, respectively; $m_{\alpha}, m_{\gamma}, m_{c m}, m_{c d}, s_{\alpha}, s_{\gamma}, s_{c m}$, $s_{c d}, r_{\alpha}, r_{\gamma}, r_{c m}$, and $r_{c d}$ are the equivalent mass, stiffness, and friction losses of diaphragms 1 and 2 , and the main and dummy cones, respectively; $s_{\beta}$ and $r_{\beta}$ are the equivalent stiffness and friction loss of the rods between diaphragms 1 and $2 ; s_{\alpha c}$ and $r_{\alpha c}$ are the equivalent stiffness and friction loss of the junction parts between diaphragm 1 and the dummy cone; $s_{\gamma c}$ and $r_{\gamma c}$ are the equivalent stiffness and

friction loss of the junction parts between diaphragm 2 and the main cone; $\zeta_{\alpha}, \zeta_{\gamma}, \zeta_{\alpha c}, \zeta_{c \alpha}, \zeta_{\gamma c}$, and $\zeta_{c \gamma}$ are the junction coefficients between diaphragms 1 and 2 , diaphragm 1 and the dummy cone, and diaphragm 2 and main cone, respectively; $F_{\alpha}$ and $F_{\gamma}$ are the exciting forces of diaphragms 1 and 2, respectively; $Z_{c m}$ and $Z_{c d}$ are the radiation impedance of the main and dummy cones, respectively.

To calculate the eigenfrequencies and eigenvectors of the vibration of the transducer shown in Figs. 2 and 3, we rewrite Eqs. (1)-(4) for a Fourier component by the matrix form. We assume that diaphragms 1 and 2, and the dummy cone and main cone are symmetric to each other, i.e.,

$$
\begin{aligned}
& m_{\alpha}=m_{\gamma}=m_{1}, \quad m_{c d}=m_{c m}=m_{2}, \quad s_{\alpha}=s_{\gamma}=s_{1}, \\
& s_{c d}=s_{c m}=s_{2}, \quad s_{\alpha c}=s_{\gamma c}=s_{\beta 2}, \quad s_{\beta}=s_{\beta 1}, \\
& \zeta_{\alpha}=\zeta_{\gamma}=\zeta_{1}, \quad \zeta_{\alpha c}=\zeta_{c \alpha}=\zeta_{\gamma c}=\zeta_{c \gamma}=\zeta_{2} .
\end{aligned}
$$

Equations (1)-(4) are rewritten for a Fourier component by the matrix form as

$$
\left[K-\omega^{2} M\right]\left(\begin{array}{c}
\Phi_{\alpha} \\
\Phi_{\gamma} \\
\Phi_{c d} \\
\Phi_{c m}
\end{array}\right)=\left(\begin{array}{c}
F_{\alpha} \\
F_{\gamma} \\
F_{c d} \\
F_{c m}
\end{array}\right),
$$

$$
K=\left(\begin{array}{cccc}
s_{1}+s_{\beta 1} \zeta_{1}+s_{\beta 2} \zeta_{2} & -s_{\beta 1} \zeta_{1} & -s_{\beta 2} \zeta_{2} & 0 \\
-s_{\beta 1} \zeta_{1} & s_{1}+s_{\beta 1} \zeta_{1}+s_{\beta 2} \zeta_{2} & 0 & -s_{\beta 2} \zeta_{2} \\
-s_{\beta 2} \zeta_{2} & 0 & s_{2}+s_{\beta 2} \zeta_{2} & 0 \\
0 & -s_{\beta 2} \zeta_{2} & 0 & s_{2}+s_{\beta 2} \zeta_{2}
\end{array}\right)
$$

and

$$
M=\left(\begin{array}{cccc}
m_{1} & 0 & 0 & 0 \\
0 & m_{1} & 0 & 0 \\
0 & 0 & m_{2} & 0 \\
0 & 0 & 0 & m_{2}
\end{array}\right)
$$

where $F_{c d}$ and $F_{c m}$ are mechanical forces caused by the friction losses and the radiation impedance of the radial cones, respectively. From Eqs. (6)-(8), four positive eigen angular frequencies $\omega_{1}, \omega_{2}, \omega_{3}$, and $\omega_{4}$ are calculated. The four resonant frequencies are divided into two groups. The first pair is $\omega_{1}$ and $\omega_{3}$, and the second pair is $\omega_{2}$ and $\omega_{4}$. When the junction coefficient of three rods $\zeta_{1}$ is small, $\omega_{1}$ becomes close to $\omega_{3}$, and $\omega_{2}$ becomes close to $\omega_{4}$. This phenomenon can be confirmed by a calculation:

$$
\lim _{\zeta_{1} \rightarrow 0}\left(\omega_{1}-\omega_{3}\right)=0, \quad \lim _{\zeta_{1} \rightarrow 0}\left(\omega_{2}-\omega_{4}\right)=0 .
$$

When the mechanical forces $F_{c d}$ and $F_{c m}$ caused by the friction losses and the radiation impedances $Z_{c d}$ and $Z_{c m}$ are negligibly small, the vibration forces exciting the eigenmodes can be simplified. The condition of $F_{c d}=F_{c m}=0$ is

$$
r_{c d}=r_{\alpha c}=Z_{c d}=r_{c m}=r_{\gamma c}=Z_{c m}=0 .
$$

When Eq. (10) is fulfilled, the vibration forces exciting four eigenmodes are expressed as

$$
\begin{aligned}
& \left(\begin{array}{c}
F_{\alpha, 1} \\
F_{\gamma, 1}
\end{array}\right)=f_{1}\left(\begin{array}{l}
1 \\
1
\end{array}\right), \quad\left(\begin{array}{l}
F_{\alpha, 2} \\
F_{\gamma, 2}
\end{array}\right)=f_{2}\left(\begin{array}{l}
1 \\
1
\end{array}\right), \\
& \left(\begin{array}{c}
F_{\alpha, 3} \\
F_{\gamma, 3}
\end{array}\right)=f_{3}\left(\begin{array}{c}
1 \\
-1
\end{array}\right), \quad\left(\begin{array}{l}
F_{\alpha, 4} \\
F_{\gamma, 4}
\end{array}\right)=f_{4}\left(\begin{array}{c}
1 \\
-1
\end{array}\right),
\end{aligned}
$$

where $f_{1}, f_{2}, f_{3}$, and $f_{4}$ are constants calculated using the circuit constants in the equivalent circuit (Fig. 3). These results of the theoretical calculation show that the resonant mode of the developed transducer with cones can be controlled by adjusting the phase difference between the two voltage inputs.

The requirements that allow for this control via the two voltage inputs are as follows.

1. Cones must be fixed tightly. The adhesive layers between diaphragms and cones must be thin to prevent an increase in the mechanical friction losses. 
2. The mechanical impedance of the radial cones must be much higher than the radiation impedance of the radial cones.

To fulfill requirement 1 , the adhered area, structure, and manufacturing process between diaphragms and cones must be carefully designed. With regard to requirement 2 , the radial cones must be fabricated from metals such as aluminum alloy, whose mechanical impedance is much higher than that of air. Generally, this requirement is usually fulfilled. On the other hand, the thickness of the radial cones must be designed considering the sound pressure level and manufacturing process.

\subsection{Detailed Design of Transducer}

The two cones shown in Fig. 2 have the same design and dimensions to satisfy the assumption of Eq. (5). To satisfy the first requirement mentioned above, the junction point of the diaphragms and the cones must be carefully designed. The cone structure consists of two parts: the cone and its support. The support is employed to expand the adhesive area. To adhere the cones to the diaphragms stably, we divided the cone structure into the cone and its support. The cones are made from duralumin A2017, which is the same material as that of the rods. Figure 4 shows the structure and dimensions of the radial cone.

Next, we explain the design point related to the thickness of the radial cones. Using finite element method (FEM) calculation, we confirmed the relationship between the cone thickness and the radiated sound pressure level. FEM calculation was executed by FEMTET (Murata Sotware Co., Ltd.). The amplitude of the input voltage to two piezoelectric elements was $5.0 \mathrm{~V}$ in rms value, and the phase difference between them was $0 \mathrm{deg}$. The physical properties of the cone, rod, and diaphragm are shown in Table 1. The physical properties of the elasto-piezodielectric matrix of the piezoelectric element are listed in Table 2.

Figure 5 shows the vibration form of the developed transducer having two cones; it was calculated by FEM. The thickness of the cone was $0.30 \mathrm{~mm}$, and the frequency was the resonant frequency of $48.0 \mathrm{kHz}$. As shown in Fig. 5, the vibrational displacement is concentrated on the

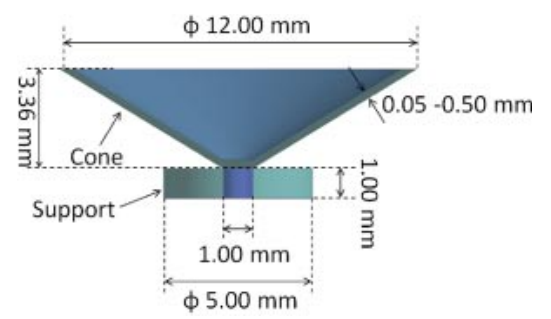

Fig. 4 Schematic and dimensions of the radial cone.
Table 1 Physical properties of the rod, cone, and diaphragm.

\begin{tabular}{cccc}
\hline Part & Material & $\begin{array}{c}\text { Mass } \\
\text { density kg } / \mathrm{m}^{3}\end{array}$ & $\begin{array}{c}\text { Young's } \\
\text { modulus GPa }\end{array}$ \\
\hline resin rod & ABS resin & $1.20 \times 10^{3}$ & 2.65 \\
\hline $\begin{array}{c}\text { metal } \\
\text { cylinder }\end{array}$ & $\begin{array}{c}\text { duralumin } \\
\text { A2017 }\end{array}$ & $2.70 \times 10^{3}$ & 69.9 \\
\hline $\begin{array}{c}\text { radial } \\
\text { cone }\end{array}$ & $\begin{array}{c}\text { duralumin } \\
\text { A2017 }\end{array}$ & $2.70 \times 10^{3}$ & 69.9 \\
\hline diaphragm & $\begin{array}{c}\text { phosphor bronze } \\
\text { C5210 }\end{array}$ & $8.80 \times 10^{3}$ & 110.0 \\
\hline
\end{tabular}

Table 2 Physical properties of the piezoelectric element: Material code C, NTK CERATEC Co., Ltd.

\begin{tabular}{cccc}
\hline $\begin{array}{c}\text { Relative dielectric } \\
\text { constant }\end{array}$ & $\begin{array}{c}\text { Coupling } \\
\text { factor }\end{array}$ & $\begin{array}{c}\text { Piezoelectric } \\
\text { strain constant } \\
\times 10^{-12} \mathrm{~m} / \mathrm{V}\end{array}$ & $\begin{array}{c}\text { Elastic } \\
\text { constant } \\
\times 10^{-12} \mathrm{~m}^{2} / \mathrm{N}\end{array}$ \\
\hline$\epsilon_{33} / \epsilon_{0}=4,500$ & $K_{r}=0.61$ & $d_{31}=-160$ & $S_{11}=15.2$ \\
$\epsilon_{11} / \epsilon_{0}=4,700$ & $K_{31}=0.35$ & $d_{33}=280$ & $S_{33}=15.5$ \\
& $K_{33}=0.65$ & $d_{15}=450$ & \\
\hline
\end{tabular}

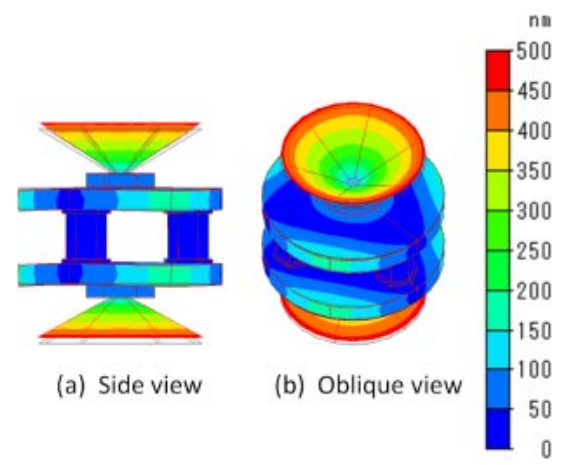

Fig. 5 Calculation result of the vibration form of the transducer having two cones at the resonant frequency of $48.0 \mathrm{kHz}$ (cone thickness $=0.30 \mathrm{~mm}$ ).

cones, particularly the edge of the cones. This calculation result shows that cones provide the displacement expansion mechanism.

Figure 6 shows the dependence of the frequency response of the sound pressure level at $20 \mathrm{~cm}$ from the top of the cone on the cone thickness. The cone thickness was varied from 0.05 to $0.50 \mathrm{~mm}$. As shown in Fig. 6, the sound pressure level increases with decreasing thickness of the cone. This is due to the increase in the vibration displacement of the cone. Note that the resonant peak shifts to the high-frequency region as the thickness of the cone decreases. We set the thickness of the cone to the minimum thickness of the actual machining process, which was $0.30 \mathrm{~mm}$, as mentioned above. 


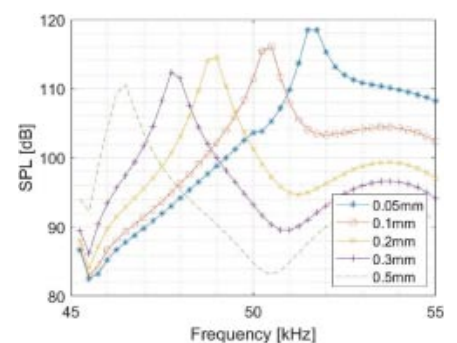

Fig. 6 Calculation results of the dependence of the frequency response of the sound pressure level at a distance of $20 \mathrm{~cm}$ from the top of the cone on cone thickness (cone thickness $=0.05 \mathrm{~mm}-0.5 \mathrm{~mm}$ ).

\section{DESIGN OF LOOP HORN}

\subsection{Basic Idea of Loop Horn}

We propose a unique horn that we named a loop horn. The loop horn is a type of sonic crystal having a periodic structure with some resonant frequencies and bandgaps.

The main goals, which are (1) to obtain narrow directivity and (2) to enhance the sound pressure of the ultrasonic wave, can be achieved using the loop horn. Sonic crystals are a type of acoustic metamaterial. There are many prior research studies on acoustic metamaterials, i.e., double-negative acoustic metamaterials, phononic crystals, and sonic crystals [15-19].

The basic idea of loop horns is based on lattice sonic crystals. An example of lattice sonic crystals and their structures are shown in Fig. 7. The target frequency was set to $40 \mathrm{kHz}$, and detailed dimensions are shown in Fig. 7. We assumed that rectangular rods are composed of perfectly rigid material. The lattice shown in Fig. 7 can be regarded as the two-dimensional array of rods of infinite length. This lattice sonic crystal has some resonant modes, which are caused by the periodic structure of the lattice. The details are shown in the Appendix.

Here, we describe the difference between the lattice sonic crystal and the loop horn shown in Fig. 8(a). There are two important points, which are related to (1) the number of columns of the sonic crystal and (2) the difference in the shape i.e., infinite rectangular lattice or circular loop.

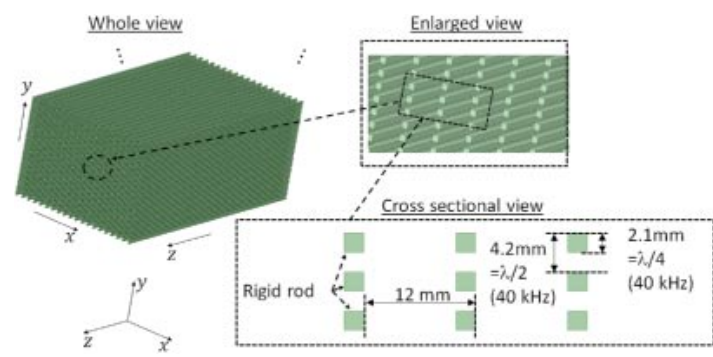

Fig. 7 Schematic of the lattice sonic crystal consisting of rectangular rods.
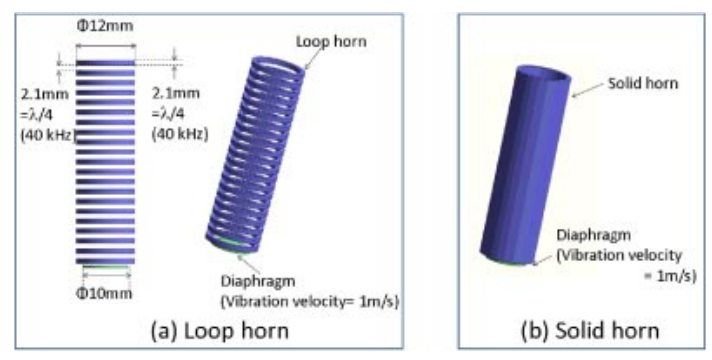

Fig. 8 Schematic of the FEM model of a circular diaphragm and the cylindrical loop horn.

First, we explain point (1) mentioned above. A reciprocal lattice is defined by the Fourier transform of a Bravais lattice. If we take out two columns of the lattice sonic crystal, the band structure shown in Fig. A.2 is retained owing to the characteristics of the Fourier transform. Thus, a lattice sonic crystal having only two columns has the same function as the lattice sonic crystal shown in Fig. 7.

Next, we explain point 2 using the Bloch's theorem. Although both the infinite lattice and the axisymmetric circular loops can be drawn in the same two-dimensional cross section, the Laplacian is different in terms of the translational symmetry. In the axisymmetric coordinate system $(r, z)$, the Laplacian is written as

$$
\nabla^{2}=\frac{\partial^{2}}{\partial r^{2}}+\frac{\partial^{2}}{\partial z^{2}}+\frac{1}{r} \frac{\partial}{\partial r}
$$

The first and second terms on the right-hand side of Eq. (12) have translational symmetry. The third term, however, does not have the translational symmetry. Thus, if the velocity potential $\phi$ satisfies $\partial \phi / \partial r=0$, i.e., the velocity potential becomes a constant in the direction of the $r$-axis, Bloch's theorem is fulfilled. Therefore, the axisymmetric loop horn acts as the sonic crystal and waveguide for the plane wave propagating in the direction of the $z$-axis. When the size of the sound source is sufficiently larger than the wavelength, the main component of the radiated velocity potential is the plane wave propagating in the direction of the $z$-axis.

Figure 8(a) shows the loop horn whose target frequency is $40 \mathrm{kHz}$. Figure 8 (b) shows the solid straight horn. At the bottom of the loop horn, there is a diaphragm with a vibration velocity of $1 \mathrm{~m} / \mathrm{s}$. Twenty loops are set above the diaphragm. The cross section of a loop of the loop horn is square. The length of the sides of the cross section and the pitch are equal to $\lambda / 4$ and $\lambda / 2$, respectively.

To confirm the behavior of the loop horn, we calculate the radiation impedance and the sound field by FEM. Figures 9(a) and 9(b) show the calculated frequency responses of the real and imaginary parts, respectively, of the radiation impedance $\left(r_{s}\right.$ and $x_{s}$ ) normalized by the specific acoustic impedance of air $\rho c$, when there is only a 

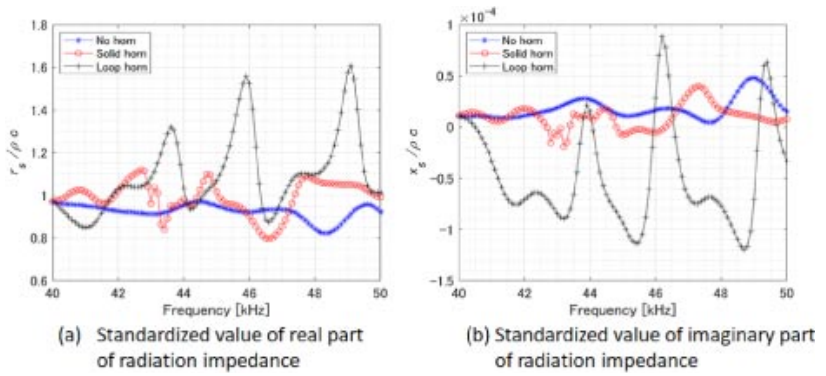

Fig. 9 Calculated frequency response of the radiation impedance (with no horn, solid horn, and loop horn).

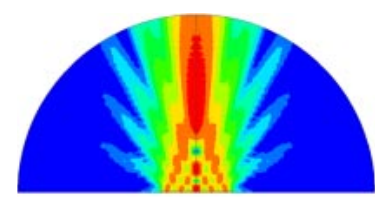

(a) No horn

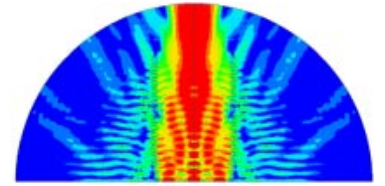

(b) Loop horn

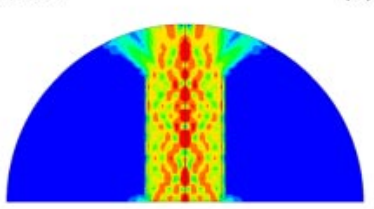

(c) Solid horn

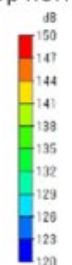

Fig. 10 Results of the FEM calculation when no horn is utilized, a loop horn is utilized, and a solid horn is utilized (Frequency $=45.9 \mathrm{kHz}$ ).

diaphragm and when the solid horn or the loop horn is utilized. $\rho$ and $c$ are the mass density of air and speed of sound in air, respectively. As shown in Fig. 9(a), when the loop horn is utilized, at certain frequencies, the real part of the radiation impedance is strongly enhanced compared with when the solid horn is used. These results show that the loop horn acts as an acoustic resonant structure and is useful for enhancing the sound pressure of the radiated ultrasonic wave.

We show the sound field obtained by FEM as contour figures. The frequency was set to the second resonant frequency of $45.9 \mathrm{kHz}$ when the loop horn was utilized. Figures 10(a), 10(b), and 10(c) show the cross sections of the contours of the sound pressure calculated by FEM. As shown in Fig. 10(a), the sound pressure is widely distributed around the diaphragm when horns are not utilized. On the other hand, as shown in Figs. 10(b) and 10(c), when the loop horn or the solid horn is set above the diaphragm, the sound pressure is concentrated in the horns. In particular, the sound pressure is sufficiently concentrated and enhanced when the loop horn is utilized.

The basic idea of the loop horn can be extended to various shapes, structures, and dimensions. Moreover, the combination of the loop horn and other types of sonic crystals can be considered. Note that the loop horn does not

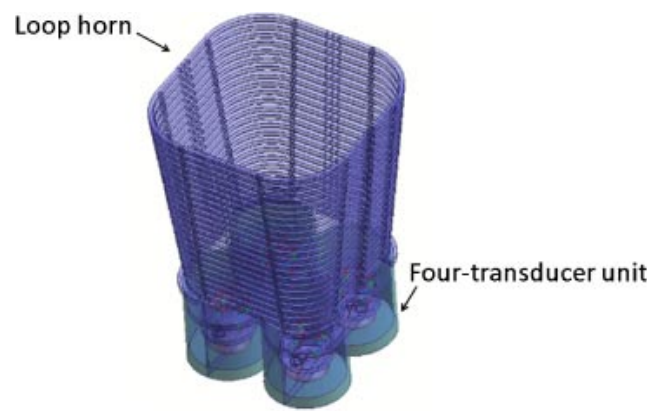

Fig. 11 Schematic of the layout of the four-transducer unit with the truncated pyramidal loop horn.

have any effect on the demodulated sounds because the thickness of each loop is much smaller than the wavelength of the audible sounds.

\subsection{Detailed Design of Loop Horn}

In this study, we fabricated the four-transducer unit shown in Fig. 11, which has four transducers and one loop horn.

There are two important design points regarding the loop horn for the four-transducer unit. The first design point is the pitch between each loop and the size of the cross section of each loop, which must be designed while considering the target frequency band. The second point is the solid angle of the loop array. The simple loop horn shown in Fig. 8(a) has a straight cylindrical shape, i.e., the cross section is constant. However, to maximize the sound pressure at a far distance from the transducer unit, the cross section of the loop horn must be continuously changed. In this paper, we consider a loop horn having a truncated pyramid shape. Thus, the important parameter is the solid angle of the pyramid shape.

First, we consider the pitch between each loop and the size of the cross section of each loop. As mentioned above, the cross section of each loop is square, and the length of one side of the cross section and the pitch are equal to $\lambda / 4$ and $\lambda / 2$, respectively.

The resonant frequencies of the developed piezoelectric transducer are from $45 \mathrm{kHz}$ to $60 \mathrm{kHz}$; thus, one-quarter of the wavelength is $1.4 \mathrm{~mm} \leq \lambda \leq 1.9 \mathrm{~mm}$. The pitch of $D / 2=1.50 \mathrm{~mm}$ was selected considering the minimum pitch of a $3 \mathrm{D}$ printer. $D$ is the vertical distance between loops.

Next, we consider the design of the solid angle of the loop horn. The solid angle $\Omega$ is calculated, using the gradient shown in Fig. 12, as $\Omega=2 \pi(1-\cos \theta)$ sr. From the FEM calculation results, we conclude that the optimum solid angle is $0.0468 \mathrm{sr}$, which corresponds to the gradient of $\theta=7 \mathrm{deg}$. Figure 13 shows the FEM calculation results of the frequency response of the sound pressure level. Figure 13 shows three curves, which represent the fre- 


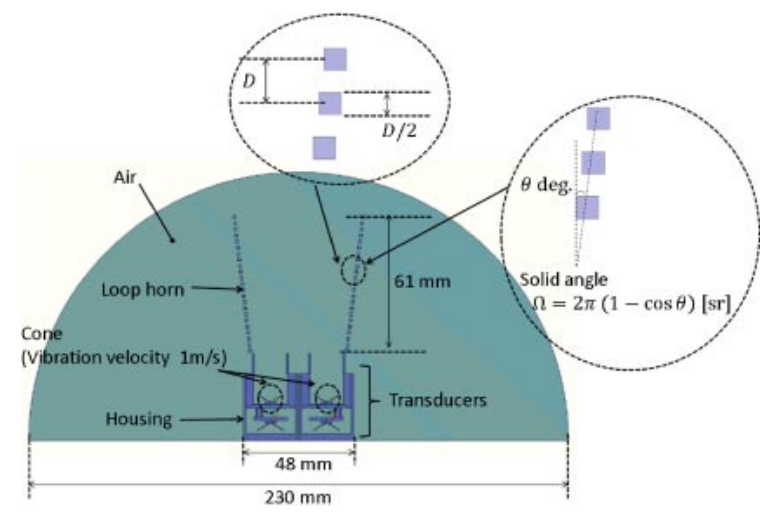

Fig. 12 Schematic of the FEM model of the fourtransducer unit with the loop horn (two-dimensional model).

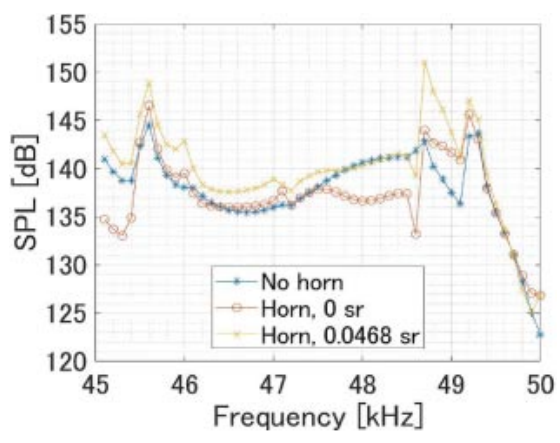

Fig. 13 Dependence of the frequency response of the sound pressure level on the solid angle of the truncated pyramidal loop horn.

quency responses of the sound pressure level at the distance of $20 \mathrm{~cm}$ when the loop horn is not utilized, the solid angle is $0 \mathrm{sr}$, and the solid angle is $0.0468 \mathrm{sr}$. As shown in Fig. 13, the sound pressure level when the solid angle is $0.0468 \mathrm{sr}$ is higher than other conditions at the resonant frequencies.

\section{EXPERIMENT}

We fabricated the piezoelectric transducer and fourtransducer unit on the basis of the detailed design described in the preceding section.

The measuring distance was decided considering shortrange voice communication. The Rayleigh distance of the four-transducer unit for the ultrasonic wave of $48 \mathrm{kHz}$ was calculated as $16 \mathrm{~cm}$ from the length of the transducer array and the wavelength of the ultrasonic wave. Thus, the measuring distance of $20 \mathrm{~cm}$ was larger than the Rayleigh distance of the four-transducer unit. On the other hand, the far field of demodulated audible sound is formed at a distance greater than several times of the Rayleigh distance. Therefore, the measuring distance of $20 \mathrm{~cm}$ was defined as the near field for the demodulated sound. For

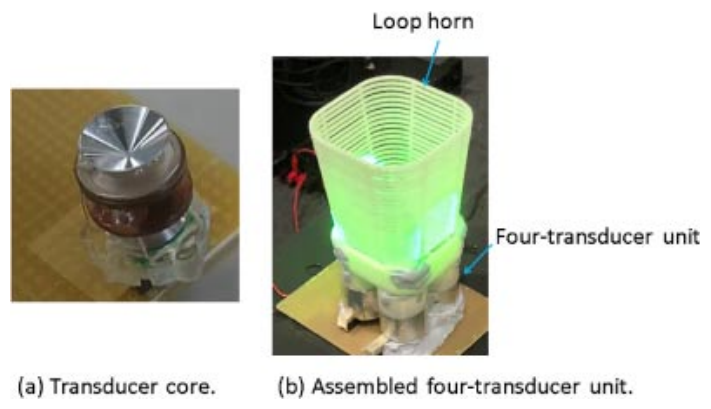

Fig. 14 Photographs of the fabricated transducer and the four-transducer unit with the loop horn.

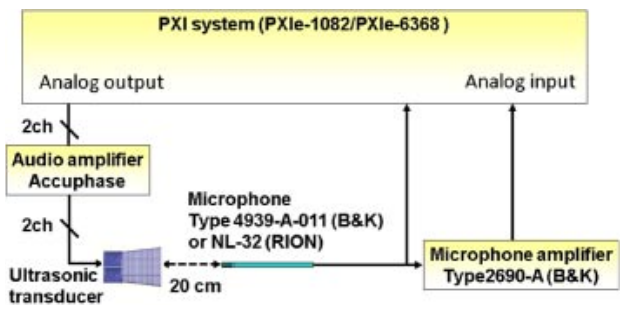

Fig. 15 Schematic of the ultrasonic and audible sound measurement system.

this reason, the measured radiation pattern of demodulated sounds was defined as the near-field radiation pattern.

\subsection{Ultrasonic Characteristics of the Four-transducer Unit}

Figures 14(a) and 14(b) show the photographs of the fabricated transducer. Figure 14(a) shows the core of the transducer and 14(b) shows the four-transducer unit with the loop horn. The transducer core is supported by three rubber parts and housed in an Acrylonitrile-Styrene-Acrylate resin (ABS resin) case.

Figure 15 shows the measurement system of the sound pressure level of the transducer.

The PXI platform (National Instruments Corporation) was employed as the central measuring system. The distance between the microphone Type 4936-A-011 and the top of the box of the transducer was $20 \mathrm{~cm}$. Experimental conditions were as follows

- Measuring system: Fig. 15

- Room: Anechoic chamber in Waseda University

- Input signal: Sweep tone $(30.0-60.0 \mathrm{kHz}, 5 \mathrm{~V}$ in rms)

- Microphone and conditioning amplifier: Type 4936-A011 and Type 2690-A, respectively

- Distance between the microphone and the top of the case cap or the top of the loop horn: $20 \mathrm{~cm}$

- Rotation of the transducer using a turntable controlled by the PXI controller and LabVIEW

Figure 16 shows the experimental data of the frequency response of the sound pressure level of the ultrasonic wave 


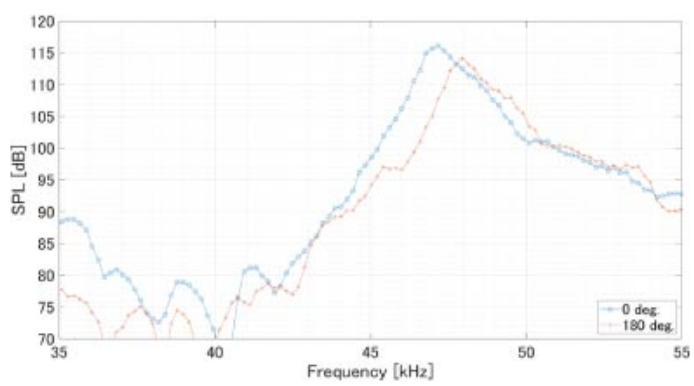

Fig. 16 Frequency response of the sound pressure level of the single transducer.

of the single transducer shown in Fig. 14(a). The resonant peaks change owing to the phase difference between the two voltage inputs of diaphragms 1 and 2 . When the two voltage inputs were in-phase and anti-phase, the resonant frequencies were $47.17 \mathrm{kHz}$ and $47.95 \mathrm{kHz}$, respectively.

Figures 17(a) and 17(b) show the directivities of the sound pressure level at the first resonant frequency of $47.17 \mathrm{kHz}$ when the phase differences between two voltage inputs are $0 \mathrm{deg}$ and $180 \mathrm{deg}$, respectively. Figures 18(a) and 18(b) show the directivities at the second resonant frequency of $47.95 \mathrm{kHz}$. As shown in Figs. 17(a) and 17(b), and Figs. 18(a) and 18(b), the directivities for the phase

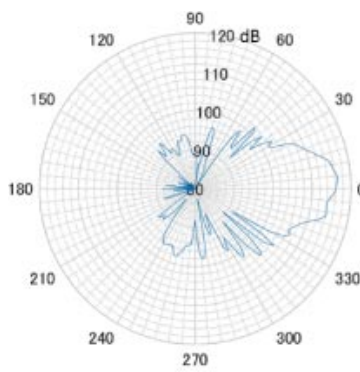

(a) Phase difference $=0 \mathrm{deg}$

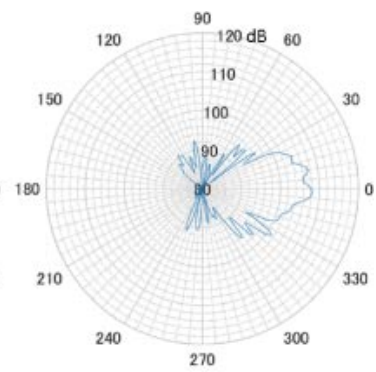

(b) Phase difference $=180 \mathrm{deg}$
Fig. 17 Directivities of the sound pressure level of one developed transducer at the first resonant frequency of $47.17 \mathrm{kHz}$.

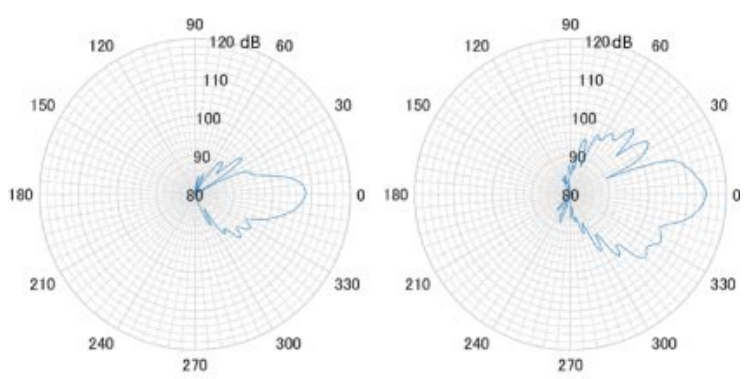

(a) Phase difference $=0$ deg

(b) Phase difference $=180 \mathrm{deg}$

Fig. 18 Directivities of the sound pressure level of one developed transducer at the second resonant frequency of $47.95 \mathrm{kHz}$.

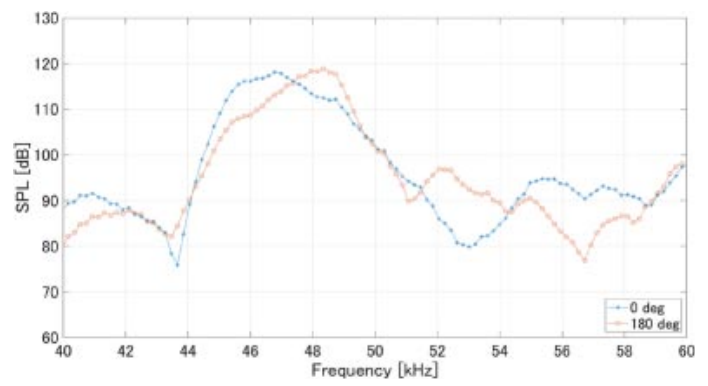

Fig. 19 Frequency responses of the sound pressure level of the four-transducer unit without the loop horn.

differences of $0 \mathrm{deg}$ and $180 \mathrm{deg}$ slightly changed. This is due to the change in the vibration form, which depends on the phase difference of the two voltage inputs.

Figure 19 shows the experimental data of the frequency response of the sound pressure level of the ultrasonic wave of the four-transducer unit shown in Fig. 14(b). As shown in Fig. 19, the control function of resonant frequencies is effective for the four-transducer unit. The resonant frequencies of $46.78 \mathrm{kHz}$ and $48.54 \mathrm{kHz}$ were excited by the phase differences of $0 \mathrm{deg}$ and $180 \mathrm{deg}$, respectively.

Figures 20(a) and 20(b), and Figs. 21(a) and 21(b) show the directivities of the sound pressure level at the

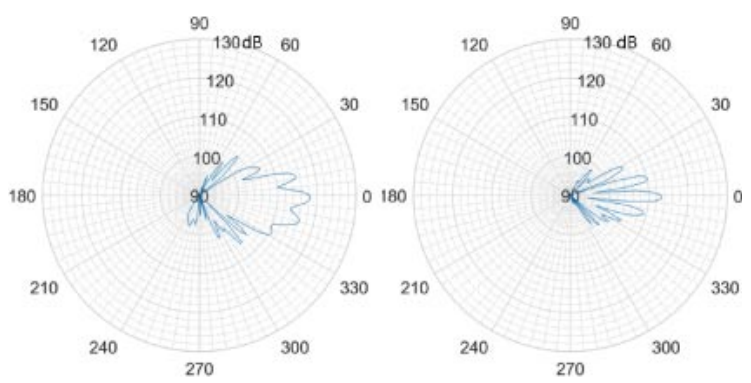

(a) Phase difference $=0$ deg

(b) Phase difference $=180 \mathrm{deg}$

Fig. 20 Directivities of the sound pressure level of a four-transducer unit without the loop horn at the first resonant frequency of $46.78 \mathrm{kHz}$.

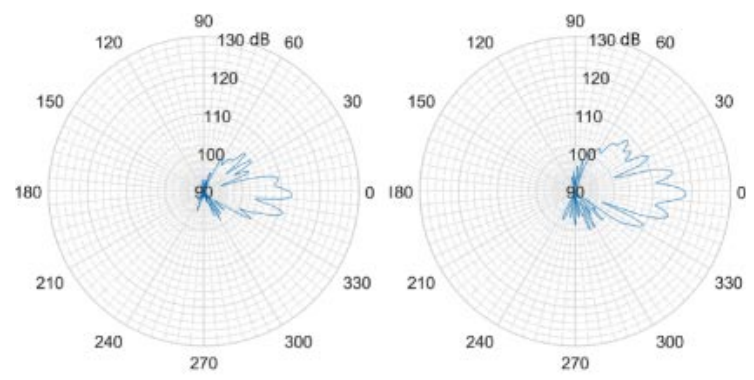

(a) Phase difference $=0$ deg

(b) Phase difference $=180 \mathrm{deg}$

Fig. 21 Directivities of the sound pressure level of a four-transducer unit without the loop horn at the second resonant frequency of $48.54 \mathrm{kHz}$. 
resonant frequencies of $46.78 \mathrm{kHz}$ and $48.54 \mathrm{kHz}$, respectively.

Figure 22 shows the experimental data of the frequency response of the sound pressure level of the ultrasonic wave of the four-transducer unit with the loop horn. The resonant peaks of $47.76 \mathrm{kHz}$ and $48.73 \mathrm{kHz}$ of the four-transducer unit with the loop horn are sharper than those shown in Fig. 19. The maximum sound pressure level at the resonant frequencies increased owing to the presence of the loop horn. The increase in the sound pressure level at the resonant frequencies was more than $6 \mathrm{~dB}$. Figures 23(a) and 23(b), and Figs. 24(a) and 24(b) show the directivities of the sound pressure level at the first and second resonant

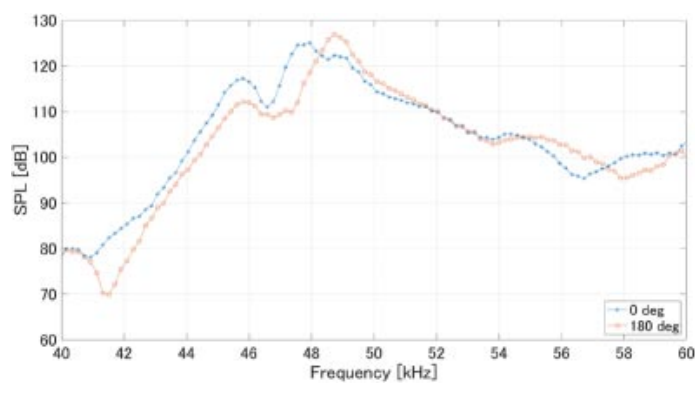

Fig. 22 Frequency responses of the sound pressure level of the four-transducer unit with the loop horn.

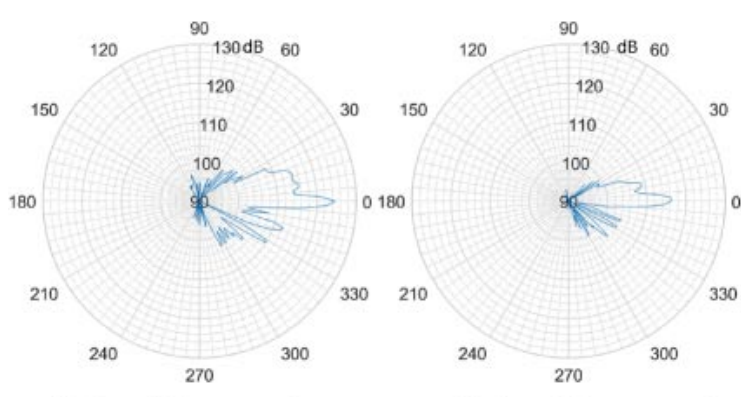

(a) Phase difference $=0 \mathrm{deg}$

(b) Phase difference $=180 \mathrm{deg}$

Fig. 23 Directivities of the sound pressure level of a four-transducer unit with the loop horn at the first resonant frequency of $47.76 \mathrm{kHz}$.

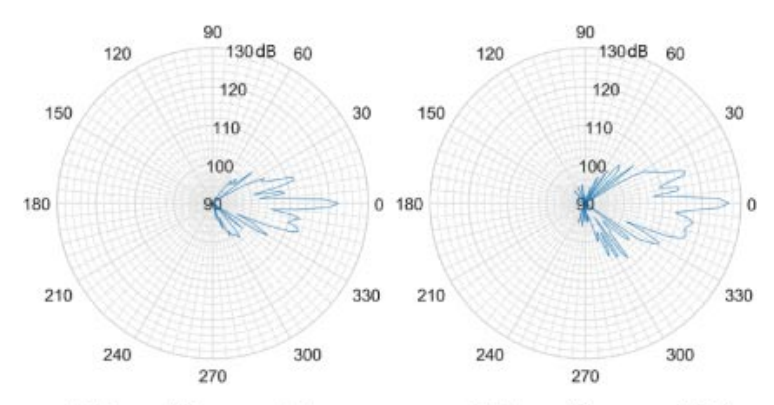

(a) Phase difference $=0 \mathrm{deg}$

(b) Phase difference $=180 \mathrm{deg}$

Fig. 24 Directivities of the sound pressure level of a four-transducer unit with the loop horn at the second resonant frequency of $48.73 \mathrm{kHz}$. frequencies of $47.76 \mathrm{kHz}$ and $48.73 \mathrm{kHz}$, respectively. By comparing the results shown in Figs. 23 and 24 with those in Figs. 20 and 21, it is clear that the directivities of the sound pressure level are sharpened and increased at the resonant frequencies by the loop horn. Owing to the narrow directivity of the single transducer and the array pitch between transducers, the main lobe of the directivity of the four-transducer unit becomes very narrow.

\subsection{Characteristics of Demodulated Audible Sound}

From the results of the ultrasonic characteristics, an enhancing effect on the demodulated sound by the loop horn is expected. The frequency response of the sound pressure level of the demodulated sound was measured, using a sound level meter, NL-32 (RION Co., Ltd.).

To precisely measure the demodulated sound, the spurious sound caused by the distortion of a microphone and next-stage electronic circuits such as a preamplifier must be eliminated. When the sound pressure of the ultrasonic wave is not smaller than the acoustic overload point of the microphone and electronic circuits, the sound pressure of the ultrasonic wave received by the microphone must be reduced using an acoustic filter or other methods [20,21]. In this paper, we employed NL-32, whose microphone head UC-53A and rear electronic system have the acoustic overload points of $150 \mathrm{~dB}$ and $130 \mathrm{~dB}$, respectively. Moreover, the upper cutoff frequency of the sensitivity of the microphone head is $20 \mathrm{kHz}$. Thus, the sensitivity of the microphone head is estimated to be decreased by more than $10 \mathrm{~dB}$ on the basis of the decay curve of $-12 \mathrm{~dB} /$ octave. From this estimation, the maximum sound pressure of ultrasonic waves of the four-transducer unit is much smaller than the acoustic overload point of the employed microphone system. Thus, we did not use any acoustic filters. However, it is difficult to eliminate spurious sound ideally. We are studying optical methods, such as the laser Doppler vibrometer and the parallel phase-shifting interferometry, to obtain a clear and precise understanding of the ultrasonic field and the demodulated sound field radiated from parametric speakers [22,23]. The measurement conditions of the demodulated audible sound are listed below

- Measurement system: Fig. 15

- Room: Anechoic chamber in Waseda University

- Carrier signal (carrier wave using first resonance) $=$ pure tone (frequency $=46.78 \mathrm{kHz}$ (without horn), $47.76 \mathrm{kHz}$ (with horn), phase difference $=0 \mathrm{deg}, 5 \mathrm{~V}$ in $\mathrm{rms}$ )

- Carrier signal (carrier wave using second resonance) = pure tone (frequency $=48.54 \mathrm{kHz}$ (without horn), 48.73 $\mathrm{kHz}$ (with horn), phase difference $=180 \mathrm{deg}, 5 \mathrm{~V}$ in rms)

- Modulated signal $=$ chirp signal $\quad$ (frequency $=$ from carrier frequency $\pm 500 \mathrm{~Hz}$ to carrier frequency $\pm 10 \mathrm{kHz}$, phase difference $=0 \mathrm{deg}$ or $180 \mathrm{deg}, 5 \mathrm{~V}$ in rms) 


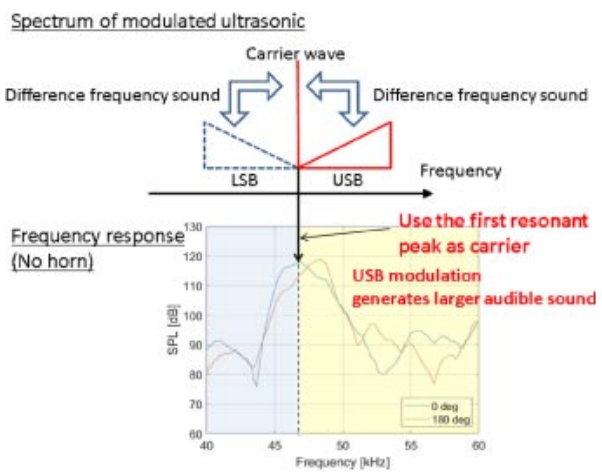

Fig. 25 Schematic of the frequency component of the modulated signal when the first resonant peak is utilized as the carrier wave.

- Modulation method: corresponding to USB (upper side band) or LSB (lower side band) modulation

- Microphone: Sound level meter NL-32

- Distance between the microphone and the top of the case cap or the top of the loop horn: $20 \mathrm{~cm}$

We explain the frequency component of the input signals to the four-transducer unit. The input signal consists of two signals: the carrier wave whose frequency $f_{c}$ corresponds to one of the resonant frequencies and the chirp signal whose frequency is from $f_{c} \pm 500 \mathrm{~Hz}$ to $f_{c} \pm 10 \mathrm{kHz}$.

Figure 25 shows the schematic of the frequency components of the input signal, i.e., the relationship between USB and LSB chirp signals, and the frequency response of the sound pressure level of the four-transducer unit (ultrasonic) shown in Fig. 19. When the first resonant frequency is utilized, the sound pressure level of the sound demodulated by the USB chirp signal is larger than that of the sound demodulated by the LSB chirp signal. In contrast, when the second resonant frequency is utilized, the sound pressure level of the demodulated sound is maximized by the LSB chirp signal.

Figures 26(a) and 26(b) show the frequency responses of the demodulated sound of the four-transducer unit when the first and second resonant frequencies are utilized and when the loop horn is utilized. As shown in Fig. 26(a), the sound pressure level of the demodulated sound was maximized when the USB chirp signal was utilized. On the other hand, Fig. 26(b) shows the frequency responses of demodulated sound when the second resonant frequencies are utilized. As shown in Fig. 26(b), the sound pressure level of the demodulated sound was maximized when the LSB chirp signal was utilized. These results show that the mode control function works well.

Finally, to clarify the effect of the loop horn, we compare the frequency responses of the demodulated sound when the loop horn is utilized and not utilized. Figure 27 shows the frequency responses of the sound pressure level
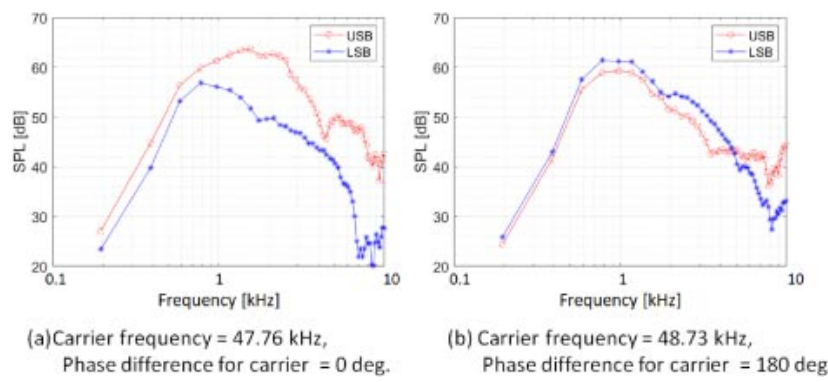

Fig. 26 Frequency responses of the sound pressure level of demodulated audible sound for a four-transducer unit with the loop horn (USB and LSB modulation). (a) Carrier frequency: $47.76 \mathrm{kHz}$ (first resonant frequency with horn), (b) carrier frequency: $48.73 \mathrm{kHz}$ (second resonant frequency with horn).

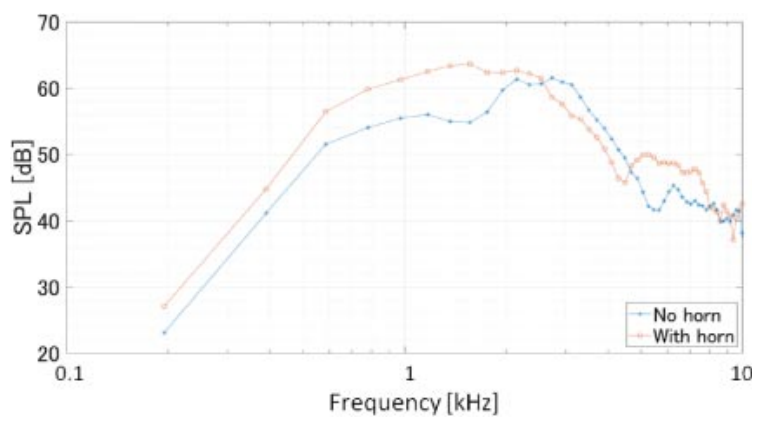

Fig. 27 Frequency responses of the sound pressure level of demodulated audible sound for a four-transducer unit with the loop horn (USB modulation, carrier frequencies are $46.78 \mathrm{kHz}$ (no horn) and $47.76 \mathrm{kHz}$ (with horn)).

of the demodulated sound when the first resonant frequencies are utilized as the carrier waves. The blue line shows the frequency response when no horn was attached to the piezoeletric transducer array. The red line shows the frequency response of the sound pressure level when the loop horn was attached to the array. As shown in Fig. 27, the sound pressure level increased from $500 \mathrm{~Hz}$ to $2.0 \mathrm{kHz}$ owing to the loop horn. The maximum sound pressure level of the demodulated sound is about $64 \mathrm{~dB}$ when the loop horn is utilized, as shown in Fig. 27. From $2.5 \mathrm{kHz}$ to $4.5 \mathrm{kHz}$, the sound pressure level of the demodulated sound is decreased by the loop horn. As shown in Fig. 22, the loop horn causes a strong resonant peak at around $48 \mathrm{kHz}$. Therefore, in some frequency bands, the sound pressure of the demodulated sound is decreased by the loop horn. To control the flatness of the demodulated sound, the loop horn having multi-resonant modes must be studied.

Figures 28 and 29 show the radiation patterns of the sound pressure level of the demodulated sound at $1.0 \mathrm{kHz}$ and $2.0 \mathrm{kHz}$, respectively. As shown in Figs. 28 and 29, the sound pressure level at frequencies lower than $2.0 \mathrm{kHz}$ is increased by the loop horn. 


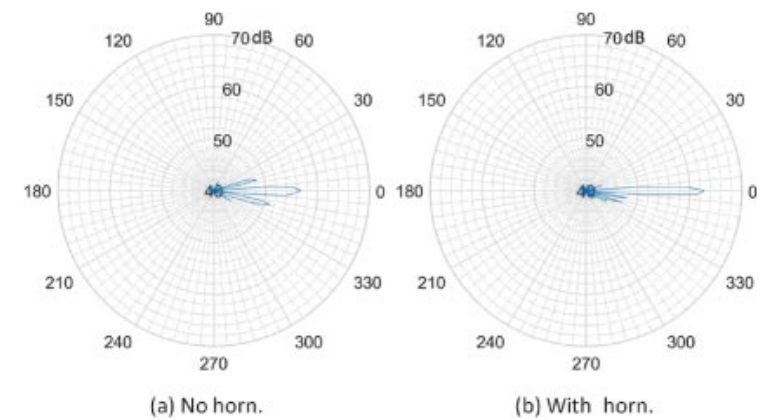

Fig. 28 Radiation patterns of the sound pressure level of demodulated audible sound at $1.0 \mathrm{kHz}$ when the loop horn is not utilized and utilized.

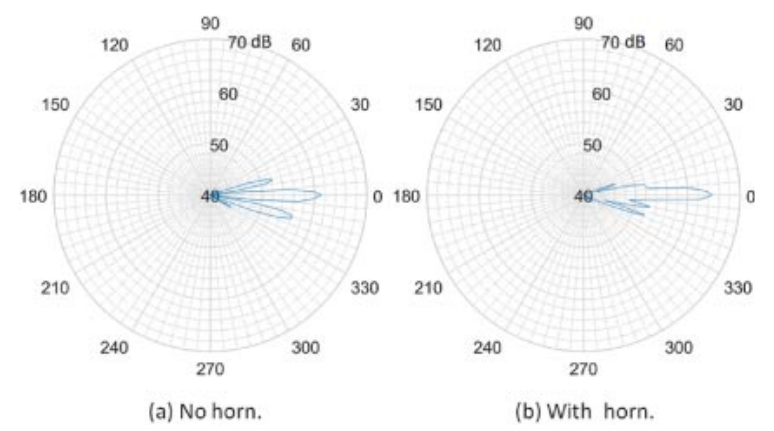

Fig. 29 Radiation patterns of the sound pressure level of demodulated audible sound at $2.0 \mathrm{kHz}$ when the loop horn is not utilized and utilized.

The causes of the narrow radiation pattern of the demodulated sound at a short-range of $20 \mathrm{~cm}$, shown in Figs. 28 and 29, can be summarized as two points, i.e., (1) the narrow directivities of the single transducer and (2) the virtual sound sources that are generated in the overlap region of the ultrasonic fields radiated from the two different vibration modes of the transducers.

\section{CONCLUSION}

In this paper, we discussed the methods used to design a parametric speaker unit consisting of a small number of transducers. The developed transducer was designed on the basis of the basic design having double-linked diaphragms and mode control function described in our previous papers. The developed small parametric speaker unit includes the peripheral acoustic structures, which are radial cones and the loop horn attached to the transducer array. It was clarified by a mathematical analysis that the two radial cones, i.e., the main and dummy cones, are necessary to maintain the function of resonant mode control.

With regard to the loop horn, first, we showed the basic idea of the loop horn based on the theoretical analysis of the lattice sonic crystal. We also showed the effect of the loop horns. With the loop horn, the sound pressure level of the ultrasonic wave increased, and the directivity became narrow. The sound pressure level of the demodulated audible sound was also increased by the loop horns at $1.0 \mathrm{kHz}$ and $2.0 \mathrm{kHz}$. The maximum sound pressure level of the sound demodulated with the input of the carrier and modulated voltage signals of $5 \mathrm{~V}$ in rms was about $64 \mathrm{~dB}$. We believe that the results and the details of the design of the ultrasonic transducer described in this paper can contribute to the fabrication of parametric speaker units consisting of a small number of transducers.

\section{REFERENCES}

[1] P. J. Westervelt, "Parametric acoustic array," J. Acoust. Soc. Am., 35, 535-537 (1963).

[2] T. G. Muir and J. G. Willete, "Parametric acoustic transmitting arrays," J. Acoust. Soc. Am., 52, 1481-1486 (1972).

[3] M. Yoneyama, J. Fujimoto, Y. Kawamo and S. Sasabe, "The audio spotlight: An application of nonlinear interaction of sound waves to a new type of loudspeaker design," J. Acoust. Soc. Am., 73, 1532-1536 (1983).

[4] T. Kamakura, M. Yoneyama and K. Ikegaya, "Studies for the realization of parametric loud speaker," J. Acoust. Soc. Jpn. $(J), 41,378-385$ (1985) (in Japanese).

[5] R. T. Beyer, "Parameter of nonlinearity in fluids," J. Acoust. Soc. Am., 32, 719-721 (1960).

[6] J. L. S. Bellin and R. T. Beyer, "Experimental investigation of an end-fire array," J. Acoust. Soc. Am., 34, 1051-1054 (1962).

[7] M. B. Moffett and R. H. Mellen, "Model for parametric acoustic sources,” J. Acoust. Soc. Am., 61, 325-337 (1977).

[8] R. L. Rolleigh, "Analysis of the broadband parametric array with Gaussian primary directivity patterns," J. Acoust. Soc. Am., 68, 649-654 (1980).

[9] N. Tanaka and M. Tanaka, "Mathematically trivial control of sound using a parametric beam focusing source," J. Acoust. Soc. Am., 129, 165-172 (2011).

[10] J. Kuroda, Y. Oikawa, Y. Yamasaki, S. Sato, M. Komoda and Y. Onishi, "Design of an ultrasonic piezoelectric transducer having double-linked diaphragms for parametric speakers," Acoust. Sci. \& Tech., 36, 385-396 (2015).

[11] J. Kuroda and Y. Oikawa, "Piezoelectric transducer with resonant modes control for parametric speaker," Acoust. Sci. \& Tech., 39, 1-10 (2018).

[12] S. Sakai, Y. Shiozawa and T. Toi, "Clarification of sound radiation mechanism for airborne ultrasonic transducer," Acoust. Sci. \& Tech., 30, 404-409 (2009).

[13] T. Irie, G. Yamada and Y. Muramoto, "Free vibration of joined conical-cylindrical shells,” J. Sound. Vib., 95, 31-39 (1984).

[14] L. Tong, "Free vibration of orthotropic conical shells," Int. J. Eng. Sci., 31, 719-733 (1993).

[15] V. G. Veselago, "The electrodynamics of substances with simultaneously negative values of $\epsilon$ and $\mu$," Sov. Phys. Usp., 10, 509-514 (1968).

[16] J. Li and C. T. Chan, "Double-negative acoustic metamaterial," Phys. Rev. E, 70, 055602 (2004).

[17] Y. Ding, Z. Liu, C. Qiu and J. Shi, "Metamaterial with simultaneously negative bulk modulus and mass density," Phys. Rev. Lett., 99, 093904 (2007).

[18] B. C. Gupta and Z. Ye, "Theoretical analysis of the focusing of acoustic waves by two-dimensional sonic crystals," Phys. Rev. $E, \mathbf{6 7}, 036603$ (2003).

[19] X. Hu, C. T. Chan and J. Zi, "Two-dimensional sonic crystals with Helmholtz resonators,” Phys. Rev. E, 71, 055601 (2005). 
[20] M. B. Bennett and D. T. Blackstock, "Parametric array in air," J. Acoust. Soc. Am., 57, 562-568 (1975).

[21] H. S. Ju and Y. H. Kim, "Near-field characteristics of the parametric loudspeaker using ultrasonic transducers," Appl. Acoust., 71, 793-800 (2010).

[22] K. Ishikawa, K. Yatabe, N. Chitanont, Y. Ikeda, Y. Oikawa, T. Onuma, H. Niwa and M. Yoshii, "High-speed imaging of sound using parallel phase-shifting interferometry," Opt. Express, 24, 12922-12932 (2016).

[23] A. T. Rosell and S. B. Figueroa, "Sound field reconstruction using acousto-optic tomography," J. Acoust. Soc. Am., 131, 3786-3793 (2012).

[24] M. S. Kushwaha, P. Halevi, G. Martinez, L. Dobrzynski and B. D. Rouhani, "Theory of acoustic band structure of periodic elastic composites," Phys. Rev. B, 49, 2313-2322 (1994).

\section{APPENDIX: BAND STRUCTURE OF LATTICE SONIC CRYSTAL}

Here, we show the behaviors of a lattice sonic crystal consisting of rectangular rods of infinite length to clarify the basic idea of the loop horn.

Figure A.1 shows the two-dimensional reciprocal lattice of the lattice sonic crystal shown in Fig. 7 in the wavenumber space. $\Gamma, X$, and $M$ are k-points in the first Brillouin zone. We calculated the band diagrams along the path of $M-\Gamma-X-M . d_{x}$ and $d_{y}$ are the lattice constants in the Bravais lattice of the sonic crystal. Figure A.2 shows the calculated band diagram of the lattice sonic crystal [24]. We employed FreeFem++ (https://freefem.org/) to calculate the sound field and to solve the matrix equation related to Bloch's theorem.

The horizontal axis of the graph in Fig. A.2 represents the reduced wavenumber along the path of $M-\Gamma-X-M$ and the vertical axis shows the frequency. As shown in Fig. $\mathrm{A} \cdot 2$, in the region of $\Gamma-X$, which corresponds to the $k_{x}$-direction, some bandgaps are formed. In these bandgaps, the propagation of the sound wave in the $x$-direction is

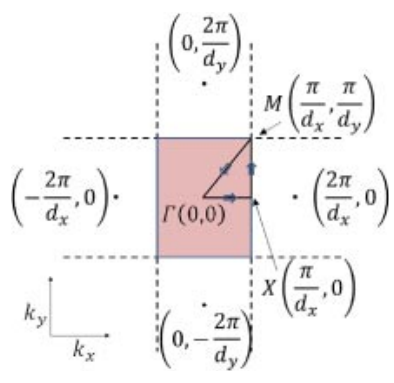

Fig. A-1 Schematic of the two-dimensional reciprocal lattice.

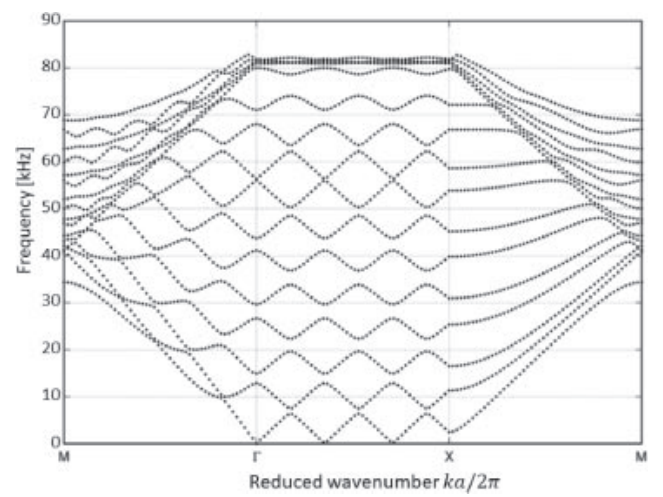

Fig. A.2 Calculated band diagram of the lattice sonic crystal consisting of rectangular rods.

prevented. On the other hand, in the region of $X-M$ corresponding to the $k_{y}$-direction, there is no bandgap. Moreover, the lines are concentrated in the frequency band from $40 \mathrm{kHz}$ to $50 \mathrm{kHz}$, which means that the density of states (DOS) is high in this frequency band. Therefore, it is expected that some resonant peaks of sound pressure due to the acoustic eigenmodes are located in this frequency band. 